\title{
Faber polynomial coefficient estimates for certain classes of bi-univalent functions defined by using the Jackson $(\mathbf{p}, \mathbf{q})$-derivative operator
}

\section{Şahsene Altınkaya*, Sibel Yalçın}

Department of Mathematics, Faculty of Arts and Science, Uludag University, 16059, Bursa, Turkey.

\author{
Communicated by B. Samet
}

\begin{abstract}
In this work, we introduce a new subclass of bi-univalent functions under the $\mathrm{D}_{\mathrm{p}, \mathrm{q}}$ operator. By using the Faber polynomial expansions, we obtain upper bounds for the coefficients of functions belonging to this analytic and bi-univalent function class. (C)2017 All rights reserved.
\end{abstract}

Keywords: Analytic functions, common fixed point, bi-univalent functions, Faber polynomials.

2010 MSC: 30C45, 33D15.

\section{Introduction, definitions, and notations}

Let $\mathcal{A}$ denote the class of functions of the form:

$$
f(z)=z+\sum_{n=2}^{\infty} a_{n} z^{n},
$$

which are analytic in the open unit disc

$$
\mathbb{U}=\{z: z \in \mathbb{C} \text { and }|z|<1\} .
$$

By $\mathcal{S}$ we denote the subclass of $\mathcal{A}$ consisting of functions of the form (1.1) which are also univalent in $\mathbb{U}$. Further, $\mathcal{P}$ be the class of functions consisting of $\varphi$, such that

$$
\varphi(z)=1+\sum_{n=1}^{\infty} \varphi_{n} z^{n},
$$

which are regular in the open unit disc $\mathbb{U}$ and satisfy the condition $\mathfrak{R}(\varphi(z))>0$ in $\mathbb{U}$. By the Carathéodory's Lemma (e.g., see [12]) we have $\left|\varphi_{n}\right| \leqslant 2$.

\footnotetext{
*Corresponding author

Email addresses: sahsene@uludag.edu.tr (Şahsene Altınkaya), syalcin@uludag.edu.tr (Sibel Yalçın)
} 
The Koebe One-Quarter Theorem [12] states that the image of $\mathbb{U}$ under every function $f$ in the normalized univalent function class $\mathcal{S}$ contains a disc of radius $\frac{1}{4}$. Thus, clearly, every such univalent function has an inverse $f^{-1}$ which satisfies the following condition:

$$
\mathrm{f}^{-1}(\mathrm{f}(z))=z \quad(z \in \mathbb{U})
$$

and

where

$$
f\left(f^{-1}(w)\right)=w \quad\left(|w|<r_{0}(f) ; \quad r_{0}(f) \geqslant \frac{1}{4}\right)
$$

$$
f^{-1}(w)=w-a_{2} w^{2}+\left(2 a_{2}^{2}-a_{3}\right) w^{3}-\left(5 a_{2}^{3}-5 a_{2} a_{3}+a_{4}\right) w^{4}+\cdots .
$$

A function $\mathrm{f} \in \mathcal{A}$ is said to be bi-univalent in $\mathbb{U}$ if both $\mathrm{f}$ and $\mathrm{f}^{-1}$ are univalent in $\mathbb{U}$. Let $\Sigma$ denote the class of bi-univalent functions defined in the unit disk $\mathbb{U}$. For a brief history of functions in the class, see [27] (see also [9, 10, 20, 22]). Recently, Srivastava et al. [27], Altınkaya and Yalçın [6], and Magesh and Yamini [21] made an effort to introduce various subclasses of the bi-univalent function class $\Sigma$ and found non-sharp coefficient estimates on the initial coefficients $\left|a_{2}\right|$ and $\left|a_{3}\right|$ (see also [28]). But the coefficient problem for each one of the following Taylor-Maclaurin coefficients

$$
\left|a_{n}\right|, \quad n \in \mathbb{N} \backslash\{1,2\} ; \quad \mathbb{N}=\{1,2,3, \cdots\}
$$

is still an open problem. In the literature, there are only a few works determining the general coefficient bounds $\left|a_{n}\right|$ for the analytic bi-univalent functions $([7,16,18])$.

The Faber polynomials introduced by Faber [13] play an important role in various areas of mathematical sciences, especially in geometric function theory. Grunsky [15] succeeded in establishing a set of conditions for a given function which are necessary and in their totality sufficient for the univalency of this function, and in these conditions the coefficients of the Faber polynomials play an important role. Schiffer [25] gave a differential equations for univalent functions solving certain extremum problems with respect to coefficients of such functions; in this differential equation appears again a polynomial which is just the derivative of a Faber polynomial (see, for details, [24]).

If the functions $f$ and $F$ are analytic in $\mathbb{U}$, then $f$ is said to be subordinate to $F$, written as

$$
f(z) \prec F(z) \quad(z \in \mathbb{U}),
$$

if there exists a Schwarz function $u(z)=\sum_{n=1}^{\infty} c_{n} z^{n}$, analytic in $\mathbb{U}$, with

$$
\mathfrak{u}(0)=0 \text { and }|\mathfrak{u}(z)|<1 \quad(z \in \mathbb{U})
$$

such that

$$
f(z)=F(u(z)) \quad(z \in \mathbb{U}) .
$$

For the Schwarz function $u(z)$ we note that $\left|c_{n}\right|<1$ (e.g., see Duren [12]).

In the field of geometric function theory, various subclasses of analytic functions have been studied from different viewpoints. The fractional q-calculus is the important tool that is used to investigate subclasses of analytic functions. Historically speaking, a firm footing of the usage of the the q-calculus in the context of geometric function theory was actually provided and the basic (or q-) hypergeometric functions were first used in geometric function theory in a book chapter by Srivastava (see, for details, [26]). In fact, the theory of univalent functions can be described by using the theory of the q-calculus. Moreover, in recent years, such q-calculus operators as the fractional q-integral and fractional q-derivative operators were used to construct several subclasses of analytic functions (see, for example, [5, 8, 23]).

For the convenience, we provide some basic definitions and concept details of q-calculus which are used in this paper. We suppose throughout the paper that $0<q<p \leqslant 1$. We recall the definitions of fractional q-calculus operators of complex-valued function $f(z)$. We shall follow the notation and terminology in [14]. 
Definition 1.1 ([11]). The ( $p, q)$-derivative of the function $f$ given by (1.1) is defined as

$$
\left(D_{p, q} f\right)(z)=\frac{f(p z)-f(q z)}{(p-q) z}, z \neq 0,
$$

and $\left(D_{p, q} f\right)(0)=f^{\prime}(0)$, provided $f^{\prime}(0)$ exists.

From Definition 1.1, we deduce that

$$
\left(D_{p, q} f\right)(z)=1+\sum_{n=2}^{\infty}[n]_{p, q} a_{n} z^{n-1}
$$

where the symbol $[n]_{p, q}$ denotes the so-called $(p, q)$-bracket or twin-basic number

$$
[n]_{p, q}=\frac{p^{n}-q^{n}}{p-q} .
$$

It happens clearly that $D_{p, q} z^{n}=[n]_{p, q} z^{n-1}$. Note also that for $p=1$, the Jackson $(p, q)$-derivative reduces to the Jackson q-derivative given by (see [17])

$$
\left(D_{q} f\right)(z)=\frac{f(z)-f(q z)}{(1-q) z}, \quad z \neq 0 .
$$

The twin-basic number is a natural generalization of the q-number, that is

$$
\lim _{\mathrm{p} \rightarrow 1}[n]_{\mathrm{p}, \mathrm{q}}=[\mathrm{n}]_{\mathrm{q}}=\frac{1-\mathrm{q}^{\mathrm{n}}}{1-\mathrm{q}}, \mathrm{q} \neq 1 .
$$

The object of this paper is to introduce a new subclasses of bi-univalent functions defined by using the Jackson $(p, q)$-derivative operator and use the Faber polynomial expansion techniques to derive bounds for the general Taylor-Maclaurin coefficients $\left|a_{n}\right|$ for the functions in this class. We also obtain estimates for the initial coefficients $\left|a_{2}\right|$ and $\left|a_{3}\right|$ of these functions.

\section{Bounds derivable by the Faber polynomial expansion techniques}

We begin this section by introducing the function class $D_{\Sigma}(p, q ; \lambda, \varphi)$ by means of the following definition.

Definition 2.1. A function $f(z)$ given by (1.1) is said to be in the class $D_{\Sigma}(p, q ; \lambda, \varphi)$ if the following subordination conditions hold true:

$$
f \in \Sigma \quad \text { and } \quad(1-\lambda) \frac{f(z)}{z}+\lambda\left(D_{p, q} f\right)(z) \prec \varphi(z), \quad(z \in \mathbb{U} ; \lambda \geqslant 1)
$$

and

$$
f \in \Sigma \quad \text { and } \quad(1-\lambda) \frac{g(w)}{w}+\lambda\left(D_{p, q} g\right)(w) \prec \varphi(w), \quad(w \in \mathbb{U} ; \lambda \geqslant 1),
$$

where $g(w)=f^{-1}(w)$.

We note from Definition 2.1 that

$$
\lim _{p \rightarrow 1-} D_{\Sigma}(p, q ; \lambda, \varphi)=\left\{f: f \in \Sigma \text { and }\left\{\begin{array}{c}
\lim _{p \rightarrow 1-}\left[(1-\lambda) \frac{f(z)}{z}+\lambda\left(D_{p, q} f\right)(z)\right] \\
\lim _{p \rightarrow 1-}\left[(1-\lambda) \frac{g(w)}{w}+\lambda\left(D_{p, q} g\right)(w)\right]
\end{array}\right\}=D_{\Sigma}(q ; \lambda, \varphi) .\right.
$$


Furthermore, we note that

$$
\lim _{q \rightarrow 1-} D_{\Sigma}(q ; \lambda, \varphi)=\left\{f: f \in \Sigma \text { and }\left\{\begin{array}{c}
\lim _{q \rightarrow 1-}\left[(1-\lambda) \frac{f(z)}{z}+\lambda\left(D_{q} f\right)(z)\right] \\
\lim _{q \rightarrow 1-}\left[(1-\lambda) \frac{g(w)}{w}+\lambda\left(D_{q} g\right)(w)\right]
\end{array}\right\}=R_{\sigma}(\lambda, \varphi),\right.
$$

where $R_{\sigma}(\lambda, \varphi)$ is the class of bi-univalent defined and studied by Kumar et al. [19].

Using the Faber polynomial expansion of functions $f \in A$ of the form (1.1), the coefficients of its inverse map $g=\mathrm{f}^{-1}$ may be expressed as follows (see [3]):

$$
g(w)=f^{-1}(w)=w+\sum_{n=2}^{\infty} \frac{1}{n} K_{n-1}^{-n}\left(a_{2}, a_{3}, \cdots\right) w^{n},
$$

where

$$
\begin{aligned}
\mathrm{K}_{n-1}^{-n}= & \frac{(-n) !}{(-2 n+1) !(n-1) !} a_{2}^{n-1}+\frac{(-n) !}{[2(-n+1)] !(n-3) !} a_{2}^{n-3} a_{3}+\frac{(-n) !}{(-2 n+3) !(n-4) !} a_{2}^{n-4} a_{4} \\
& +\frac{(-n) !}{[2(-n+2)] !(n-5) !} a_{2}^{n-5}\left[a_{5}+(-n+2) a_{3}^{2}\right] \\
& +\frac{(-n) !}{(-2 n+5) !(n-6) !} a_{2}^{n-6}\left[a_{6}+(-2 n+5) a_{3} a_{4}\right]+\sum_{j \geqslant 7} a_{2}^{n-j} V_{j},
\end{aligned}
$$

such that $V_{j}(7 \leqslant j \leqslant n)$ is a homogeneous polynomial in the variables $a_{2}, a_{3}, \cdots, a_{n}$ (see, for details, [4]). In particular, the first three terms of $\mathrm{K}_{n-1}^{-n}$ are given below:

$$
\frac{1}{2} K_{1}^{-2}=-a_{2}, \quad \frac{1}{3} K_{2}^{-3}=2 a_{2}^{2}-a_{3}, \quad \frac{1}{4} K_{3}^{-4}=-\left(5 a_{2}^{3}-5 a_{2} a_{3}+a_{4}\right) .
$$

In general, an expansion of $\mathrm{K}_{\mathrm{n}}^{\mathrm{p}}$ is given by (see [3])

$$
K_{n}^{p}=p a_{n}+\frac{p(p-1)}{2} E_{n}^{2}+\frac{p !}{(p-3) ! 3 !} E_{n}^{3}+\cdots+\frac{p !}{(p-n) ! n !} E_{n}^{n}, \quad(p \in \mathbb{Z}),
$$

where

$$
\mathbb{Z}=\{0, \pm 1, \pm 2, \cdots\} \quad \text { and } \quad E_{\mathfrak{n}}^{p}=E_{\mathfrak{n}}^{p}\left(a_{2}, a_{3}, \cdots\right)
$$

and, alternatively, by (see [1] and [2])

$$
E_{n}^{m}\left(a_{1}, a_{2}, \cdots, a_{n}\right)=\sum_{m=1}^{\infty} \frac{m !\left(a_{1}\right)^{\mu_{1}} \cdots\left(a_{n}\right)^{\mu_{n}}}{\mu_{1} ! \cdots \mu_{n} !},
$$

while $a_{1}=1$, and the sum is taken over all nonnegative integers $\mu_{1}, \cdots, \mu_{n}$ satisfying the following conditions:

$$
\mu_{1}+\mu_{2}+\cdots+\mu_{n}=m, \mu_{1}+2 \mu_{2}+\cdots+n \mu_{n}=n
$$

Evidently,

$$
E_{n}^{n}\left(a_{1}, a_{2}, \cdots, a_{n}\right)=a_{1}^{n} .
$$

Our first main result is given by Theorem 2.2 below.

Theorem 2.2. Let $\mathrm{f}$ given by (1.1) be in the class $\mathrm{D}_{\Sigma}(\mathrm{p}, \mathrm{q} ; \lambda, \varphi)(\lambda \geqslant 1)$. If $\mathrm{a}_{\mathrm{m}}=0$ for $2 \leqslant \mathrm{~m} \leqslant \mathrm{n}-1$, then

$$
\left|a_{n}\right| \leqslant \frac{2}{\left|1+\left([n]_{p, q}-1\right) \lambda\right|^{\prime}}, \quad(n \geqslant 4) .
$$


Proof. For analytic functions $f$ of the form (1.1), we have

$$
(1-\lambda) \frac{f(z)}{z}+\lambda\left(D_{p, q} f\right)(z)=1+\sum_{n=2}^{\infty}\left[1+\left([n]_{p, q}-1\right) \lambda\right] a_{n} z^{n-1}
$$

and

$$
\begin{aligned}
(1-\lambda) \frac{g(w)}{w}+\lambda\left(D_{p, q} g\right)(w) & =1+\sum_{n=1}^{\infty}\left[1+\left([n]_{p, q}-1\right) \lambda\right] b_{n} w^{n-1} \\
& =1+\sum_{n=1}^{\infty}\left[1+\left([n]_{p, q}-1\right) \lambda\right] \times \frac{1}{n} K_{n-1}^{-n}\left(a_{2}, a_{3}, \cdots, a_{n}\right) w^{n-1}
\end{aligned}
$$

On the other hand, the inequalities (2.1) and (2.2) imply the existence of two Schwartz functions $u(z)=\sum_{n=1}^{\infty} c_{n} z^{n}$ and $v(w)=\sum_{n=1}^{\infty} d_{n} w^{n}$ so that

$$
(1-\lambda) \frac{f(z)}{z}+\lambda\left(D_{p, q} f\right)(z)=\varphi(u(z))
$$

and

$$
(1-\lambda) \frac{g(w)}{w}+\lambda\left(D_{p, q} g\right)(w)=\varphi(v(w))
$$

where

$$
\varphi(u(z))=1+\sum_{n=1}^{\infty} \sum_{k=1}^{n} \varphi_{k} E_{n}^{k}\left(c_{1}, c_{2}, \cdots, c_{n}\right) z^{n}
$$

and

$$
\varphi(v(w))=1+\sum_{n=1}^{\infty} \sum_{k=1}^{n} \varphi_{k} E_{n}^{k}\left(d_{1}, d_{2}, \cdots, d_{n}\right) w^{n} .
$$

Thus, from (2.3), (2.5), and (2.7) we have

$$
\left[1+\left([n]_{p, q}-1\right) \lambda\right] a_{n}=\sum_{k=1}^{n-1} \varphi_{k} E_{n-1}^{k}\left(c_{1}, c_{2}, \cdots, c_{n-1}\right), \quad(n \geqslant 2) .
$$

Similarly, by using (2.4), (2.6), and (2.8), we find that

$$
\left[1+\left([n]_{p, q}-1\right) \lambda\right] b_{n}=\sum_{k=1}^{n-1} \varphi_{k} E_{n-1}^{k}\left(d_{1}, d_{2}, \cdots, d_{n-1}\right), \quad(n \geqslant 2) .
$$

We note that, for $a_{m}=0(2 \leqslant m \leqslant n-1)$, we have

$$
b_{n}=-a_{n}
$$

and so

$$
\left[1+\left([n]_{p, q}-1\right) \lambda\right] a_{n}=\varphi_{1} c_{n-1}, \quad-\left[1+\left([n]_{p, q}-1\right) \lambda\right] a_{n}=\varphi_{1} d_{n-1} .
$$

Now taking the absolute values of either of the above two equations and using the facts that $\left|\varphi_{1}\right| \leqslant 2$, $\left|c_{n-1}\right| \leqslant 1$, and $\left|d_{n-1}\right| \leqslant 1$, we obtain

$$
\left|a_{n}\right|=\frac{\left|\varphi_{1} c_{n-1}\right|}{\left|1+\left([n]_{p, q}-1\right) \lambda\right|}=\frac{\left|\varphi_{1} d_{n-1}\right|}{\left|1+\left([n]_{p, q}-1\right) \lambda\right|} \leqslant \frac{2}{\left|1+\left([n]_{p, q}-1\right) \lambda\right|},
$$

which evidently completes the proof of Theorem 2.2. 
By letting $p \rightarrow 1$ in Theorem 2.2, we obtain the following consequence.

Corollary 2.3. Let $\mathrm{f}$ given by (1.1) be in the class $\mathrm{D}_{\Sigma}(\mathrm{q} ; \lambda, \varphi)(\lambda \geqslant 1)$. If $\mathrm{a}_{\mathrm{m}}=0$ for $2 \leqslant \mathrm{~m} \leqslant \mathrm{n}-1$, then

$$
\left|a_{n}\right| \leqslant \frac{2(1-q)}{1-q+\left(q-q^{n}\right) \lambda}, \quad(n \geqslant 4) .
$$

\section{Estimates for the initial coefficients $a_{2}$ and $a_{3}$}

In this section, we choose to relax the coefficient restrictions imposed in Theorem 2.2 and derive the resulting estimates for the initial coefficients $a_{2}$ and $a_{3}$ of functions $f \in D_{\Sigma}(p, q ; \lambda$, $\varphi)$; given by the Taylor-Maclaurin series expansion (1.1).

Theorem 3.1. Let $f$ given by (1.1) be in the class $\mathrm{D}_{\Sigma}(\mathrm{p}, \mathrm{q} ; \lambda, \varphi)(\lambda \geqslant 1)$. Then

$$
\begin{aligned}
& \left|a_{2}\right| \leqslant \min \left\{\frac{2}{|1+(p+q-1) \lambda|}, \frac{2}{\sqrt{\left|1+\left(p^{2}+p q+q^{2}-1\right) \lambda\right|}}\right\}, \\
& \left|a_{3}\right| \leqslant \min \left\{\frac{4}{[1+(p+q-1) \lambda]^{2}}+\frac{2}{\left|1+\left(p^{2}+p q+q^{2}-1\right) \lambda\right|}, \frac{6}{\left|1+\left(p^{2}+p q+q^{2}-1\right) \lambda\right|}\right\},
\end{aligned}
$$

and

$$
\left|a_{3}-2 a_{2}^{2}\right| \leqslant \frac{4}{\left|1+\left(p^{2}+p q+q^{2}-1\right) \lambda\right|} .
$$

Proof. Replacing $n$ by 2 and 3 in (2.9) and (2.10), respectively, we find that

$$
\begin{aligned}
& {\left[1+\left([2]_{p, q}-1\right) \lambda\right] a_{2}=\varphi_{1} c_{1}, } \\
& {\left[1+\left([3]_{p, q}-1\right) \lambda\right] a_{3}=\varphi_{1} c_{2}+\varphi_{2} c_{1}^{2} } \\
- & {\left[1+\left([2]_{p, q}-1\right) \lambda\right] a_{2}=\varphi_{1} d_{1} }
\end{aligned}
$$

and

$$
\left[1+\left([3]_{p, q}-1\right) \lambda\right]\left(2 a_{2}^{2}-a_{3}\right)=\varphi_{1} d_{2}+\varphi_{2} d_{1}^{2}
$$

From (3.1) and (3.3) we obtain

$$
\mathrm{d}_{1}=-\mathrm{c}_{1}
$$

and

$$
\left|a_{2}\right|=\frac{\left|\varphi_{1} c_{1}\right|}{\left|1+\left([2]_{p, q}-1\right) \lambda\right|}=\frac{\left|\varphi_{1} d_{1}\right|}{\left|1+\left([2]_{p, q}-1\right) \lambda\right|} \leqslant \frac{2}{|1+(p+q-1) \lambda|} .
$$

Now, by adding (3.2) to (3.4), we have

$$
2\left[1+\left([3]_{p, q}-1\right) \lambda\right] a_{2}^{2}=\varphi_{1}\left(c_{2}+d_{2}\right)+\varphi_{2}\left(c_{1}^{2}+d_{1}^{2}\right)
$$

or, equivalently,

$$
\left|a_{2}\right| \leqslant \frac{2}{\sqrt{\left|1+\left(p^{2}+p q+q^{2}-1\right) \lambda\right|}} .
$$

Next, in order to find the bound on the coefficient $\left|a_{3}\right|$, we subtract (3.4) from (3.2). Thus, we get

$$
2\left[1+\left([3]_{p, q}-1\right) \lambda\right]\left(a_{3}-a_{2}^{2}\right)=\varphi_{1}\left(c_{2}-d_{2}\right)+\varphi_{2}\left(c_{1}^{2}-d_{1}^{2}\right)
$$


or

$$
\left|\mathrm{a}_{3}\right| \leqslant\left|\mathrm{a}_{2}\right|^{2}+\frac{\left|\varphi_{1}\left(\mathrm{c}_{2}-\mathrm{d}_{2}\right)\right|}{2\left|1+\left([3]_{\mathrm{p}, \mathrm{q}}-1\right) \lambda\right|} .
$$

Upon substituting the value of $a_{2}^{2}$ from (3.5) and (3.6) into (3.7), it follows that

$$
\left|a_{3}\right| \leqslant \frac{4}{[1+(p+q-1) \lambda]^{2}}+\frac{2}{\left|1+\left(p^{2}+p q+q^{2}-1\right) \lambda\right|}
$$

and

$$
\left|a_{3}\right| \leqslant \frac{6}{\left|1+\left(p^{2}+p q+q^{2}-1\right) \lambda\right|} .
$$

Finally, from (3.4), we deduce (by the Carathéodory Lemma) that

$$
\left|a_{3}-2 a_{2}^{2}\right|=\frac{\left|\varphi_{1} d_{2}+\varphi_{2} d_{1}^{2}\right|}{\left|1+\left([3]_{p, q}-1\right) \lambda\right|} \leqslant \frac{4}{\left|1+\left(p^{2}+p q+q^{2}-1\right) \lambda\right|} .
$$

This evidently completes the proof of the above theorem.

By letting $p \rightarrow 1$ in Theorem 3.1, we obtain the following consequence.

Corollary 3.2. Let $f$ given by (1.1) be in the class $D_{\Sigma}(q ; \lambda, \varphi)(\lambda \geqslant 1)$. If $a_{m}=0$ for $2 \leqslant m \leqslant n-1$, then

$$
\left|a_{2}\right| \leqslant \frac{2}{1+q \lambda}, \quad\left|a_{3}\right| \leqslant \frac{4}{(1+q \lambda)^{2}}+\frac{2}{1+\left(q^{2}+q\right) \lambda}, \quad \text { and } \quad\left|a_{3}-2 a_{2}^{2}\right| \leqslant \frac{4}{1+\left(q^{2}+q\right) \lambda} .
$$

Corollary 3.3. Let $\mathrm{f}$ given by (1.1) be in the class $\mathrm{R}_{\sigma}(\lambda, \varphi)(\lambda \geqslant 1)$. If $\mathrm{a}_{\mathrm{m}}=0$ for $2 \leqslant \mathrm{~m} \leqslant \mathrm{n}-1$, then

$$
\left|a_{2}\right| \leqslant \frac{2}{1+\lambda}
$$

and

$$
\left|\mathrm{a}_{3}\right| \leqslant \frac{4}{(1+\lambda)^{2}}+\frac{2}{1+2 \lambda} .
$$

Remark 3.4. The above estimate for $\left|\mathrm{a}_{2}\right|$ shows that the inequality (3.8) is an improvement of the estimate obtained by Kumar et al. ([19], Theorem 2.2).

\section{References}

[1] H. Airault, Symmetric sums associated to the factorization of Grunsky coefficients,Groups and symmetries, CRM Proc. Lecture Notes Amer. Math. Soc., Providence, RI, 47 (2007), 3-16. 2

[2] H. Airault, Remarks on Faber polynomials, Int. Math. Forum, 3 (2008), 449-456. 2

[3] H. Airault, H. Bouali, Differential calculus on the Faber polynomials, Bull. Sci. Math., 130 (2006), 179-222. 2

[4] H. Airault, J.-G. Ren, An algebra of differential operators and generating functions on the set of univalent functions, Bull. Sci. Math., 126 (2002), 343-367. 2

[5] A. Akgül, A new subclass of meromorphic functions defined by Hilbert space operator, Honam Math. J., 38 (2016), 495-506. 1

[6] Ş. Altınkaya, S. Yalçın, Initial coefficient bounds for a general class of biunivalent functions, Int. J. Anal., 2014 (2014), 4 pages. 1

[7] Ş. Altınkaya, S. Yalçın, Coefficient bounds for a subclass of bi-univalent functions, TWMS J. Pure Appl. Math., 6 (2015), 180-185. 1

[8] M. Aydog̃an, Y. Kahramaner, Y. Polatog̃lu, Close-to-convex functions defined by fractional operator, Appl. Math. Sci. (Ruse), 7 (2013), 2769-2775. 1

[9] D. A. Brannan (ed.), J. Clunie (ed.), Aspects of contemporary complex analysis, Proceedings of the NATO Advanced Study Institute held at the University of Durham, Durham, July 120, (1979), Academic Press, Inc. [Harcourt Brace Jovanovich, Publishers], London-New York, (1980). 1 
[10] D. A. Brannan, T. S. Taha, On some classes of bi-univalent functions, Studia Univ. Babeş-Bolyai Math., 31 (1986), 70-77. 1

[11] R. Chakrabarti, R. Jagannathan, A (p, q)-oscillator realization of two-parameter quantum algebras, J. Phys. A, 24 (1991), L711-L718. 1.1

[12] P. L. Duren, Univalent functions, Grundlehren der Mathematischen Wissenschaften [Fundamental Principles of Mathematical Sciences], Springer-Verlag, New York, (1983). 1

[13] G. Faber, Über polynomische Entwickelungen, (German) Math. Ann., 57 (1903), 1569-1573. 1

[14] G. Gasper, M. Rahman, Basic hypergeometric series, With a foreword by Richard Askey, Encyclopedia of Mathematics and its Applications, Cambridge University Press, Cambridge, (1990). 1

[15] H. Grunsky, Koeffizientenbedingungen für schlicht abbildende meromorphe Funktionen, (German) Math. Z., 45 (1939), 29-61. 1

[16] S. G. Hamidi, J. M. Jahangiri, Faber polynomial coefficient estimates for analytic bi-close-to-convex functions, C. R. Math. Acad. Sci. Paris, 352 (2014), 17-20. 1

[17] F. H. Jackson, On q-functions and a certain difference operator, Trans. Roy. Soc. Edinburgh, 46 (1908), 253-281. 1

[18] J. M. Jahangiri, S. G. Hamidi, Coefficient estimates for certain classes of bi-univalent functions, Int. J. Math. Math. Sci., 2013 (2013), 4 pages. 1

[19] S. S. Kumar, V. Kumar, V. Ravichandran, Estimates for the initial coefficients of bi-univalent functions, Tamsui Oxf. J. Inf. Math. Sci., 29 (2013), 487-504. 2, 3.4

[20] M. Lewin, On a coefficient problem for bi-univalent functions, Proc. Amer. Math. Soc. 18 (1967), 63-68. 1

[21] N. Magesh, J. Yamini, Coefficient bounds for certain subclasses of bi-univalent functions, Int. Math. Forum, 8 (2013), 1337-1344. 1

[22] E. Netanyahu, The minimal distance of the image boundary from the origin and the second coefficient of a univalent function in $z<1$, Arch. Rational Mech. Anal., 32 (1969), 100-112. 1

[23] Y. Polatoğlu, Growth and distortion theorems for generalized q-starlike functions, Adv. Math. Sci. J., 5 (2016), 7-12. 1

[24] A. C. Schaeffer, D. C. Spencer, The coefficients of schlicht functions, Duke Math. J., 10 (1943), 611-635. 1

[25] M. Schiffer, A method of variation within the family of simple functions, Proc. London Math. Soc., 2 (1938), 432-449. 1

[26] H. M. Srivastava, Univalent functions, fractional calculus, and associated generalized hypergeometric functions, Univalent functions, fractional calculus, and their applications, Kōriyama, (1988), Ellis Horwood Ser. Math. Appl., Horwood, Chichester, (1989), 329-354. 1

[27] H. M. Srivastava, A. K. Mishra, P. Gochhayat, Certain subclasses of analytic and bi-univalent functions, Appl. Math. Lett., 23 (2010), 1188-1192. 1

[28] Q.-H. Xu, Y.-C. Gui, H. M. Srivastava, Coefficient estimates for a certain subclass of analytic and bi-univalent functions, Appl. Math. Lett., 25 (2012), 990-994. 1 